\title{
A REMOTE MONITORING SYSTEM FOR A THREE-PHASE 10-KVA SWITCHABLE DISTRIBUTION TRANSFORMER USING ZIGBEE WIRELESS NETWORK
}

\author{
S.Dinesh Kumar ${ }^{1}$, R.Suresh Kumar ${ }^{2}$, Dr.Rani Thottungal ${ }^{3}$ \\ ${ }^{1}$ PG Student, EEE Department, Kumaraguru College of Technology, Tamil Nadu, India \\ ${ }^{2}$ Asst.Professor, EEE Department, Kumaraguru College of Technology, Tamil Nadu, India \\ ${ }^{3}$ Professor and Head, EEE Department, Kumaraguru College of Technology, Tamil Nadu, India
}

\begin{abstract}
A Remote Monitoring System plays an important role in many applications. This paper proposes an innovative design about the development of remote monitoring system for a three-phase 10-kVA Switchable Distribution transformer with the help of a PIC microcontroller and Zigbee-based Wireless device. The PIC microcontroller from microchip helps in controlling the switching devices and monitoring the current, voltage and temperature of the transformer. The Zigbee-based wireless device is used as long range wireless communication between the modules. The information is transmitted point by point using Zigbee transmitter and receiver and is sent to the server module to check the state of the transformer. It has a data rate speed of $250 \mathrm{Kbps}$ with minimum power consumption and has a transmission distances range from 10 to 100m. The data transmission rate is higher than other wireless systems. This is user friendly and can be achieved at low cost. This handles the transfer of parameters such as voltage, current and temperature as digital data from current transducers, voltage transformers and from temperature sensors to the server module through wireless communication. This system helps in reading sensor, controlling switches, monitoring and data protection.
\end{abstract}

Key Words: Switchable Transformer, PIC Microcontroller and Zigbee

\section{INTRODUCTION}

The Switchable distribution transformer was introduced in the year 2000. This transformer similarly works like conventional transformer but only main difference is that the use of the switching devices to connect the tappings of the primary and secondary windings of the transformer. If the switching devices implemented, the new difficulty arises such as how to control the switching devices, how to reduce the copper and core losses of the transformer and how to monitor the parameters without the displacement of the crew. Reference [1] monitors the parameters with the help of local area network and used an Atmel with built in CAN controller for communication between modules and used the other controller to perform averaging and FFT calculation [3].this further enhances wiring for communication between the modules and more controller makes the entire system more complex and used Ethernet as a wired network for remote monitoring. Uses of more controllers will make costs more to the entire System and it will also make more difficulties for the operator to identify the problems in the complex system.

As a solution to this Problem, some advantages can be done through the use of this approach in the system i.e.

1. Monitoring purpose can be carried out at the desirable range without the displacement of the crew.

2. Complication in the system can be reduced by using the single microcontroller and wireless network.

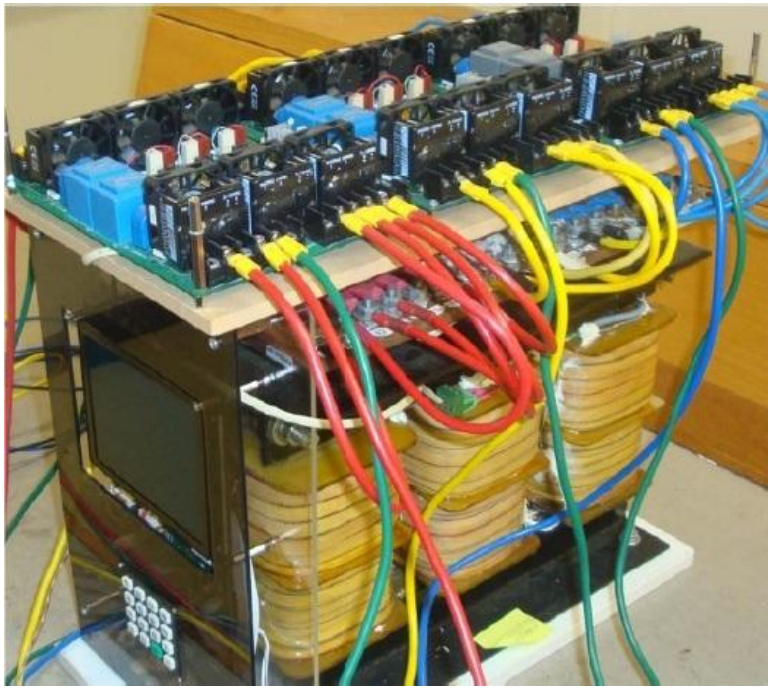

Fig-1: Three-Phase 10-kVA Switchable Transformer

3. The system costs can be reduced effectively.

In the system, a wireless device called Zigbee is used as a long range wireless communication between the modules and has data protection at the desirable range within the modules. It can be achieved at low cost and helps in long battery life in the process. This handles the transfer of parameters to the server module using Zigbee transmitter and receiver. It is a standard-based technology for remote monitoring and 
control. It supports low data rate, low power consumption, security and reliability. It allows nodes to find new routes throughout the network if one route fails, making it a robust wireless solution.

\section{HARDWARE DESCRIPTION}

\subsection{PIC16F877A MicroconTroller}

The PIC is a family of Harvard architecture microcontrollers made by Microchip Technology, derived from the PIC1640. The name PIC initially referred to "Programmable Interface Controller".

PICs are popular with both industrial developers and hobbyists alike due to their low cost, wide availability, large user base, extensive collection of application notes, availability of low cost or free development tools, and serial programming (and re-programming with flash memory) capability.

It is 8-bit microcontroller compatible with any other PIC devices. It features $200 \mathrm{~ns}$ instruction execution, 256 bytes of EEPROM data memory, self programming, an ICD, 2 Comparators, 8 channels of 10-bit Analog-to-Digital(A/D) converter, 2 capture/compare/PWM functions, synchronous serial port that can be configured as either 3-wire SPI or 2wire I2C bus, a USART, and a Parallel Slave port.

Its Features are:

- High-Performance RISC CPU

- Operating speed: $20 \mathrm{MHz}$

- Operating voltage: $4.0-5.5 \mathrm{~V}$

- Industrial temperature range $\left(-40^{\circ}\right.$ to $\left.+85^{\circ} \mathrm{C}\right)$

- 15 Interrupt Sources

- 35 single-word instructions

- 10 bit, 8 channel A/D converter

- Brown out reset

- Power saving sleep mode

- Programmable code protection

- All single-cycle instructions except for program Branches (two-cycle)

\subsection{Zigbee- based Wireless device}

Zigbee is based on IEEE 802.15 standard used to create a personal area network built from low power digital radios. It often transmits data over longer distances by passing data through intermediate devices to reach more distant ones. It targeted at radio frequency applications that require long battery life, low data rate and secure networking. Zigbee has a data rate speed of $250 \mathrm{kbps}$ best suited for single signal transmission from an input device or sensor. Transmission distances range from 10 to 100 meters line of sight, depending upon power output and environmental characteristics. The Zigbee specification is intended to be simpler and less expensive than other WPANs, such as
Bluetooth. It is a low cost, low power wireless mesh standard. The low cost allows the technology to be widely used in wireless control and monitoring system applications.

Zigbee networks are secured by 128 bit symmetric encryption keys and mesh networking in the Zigbee network provides high reliability and a more extensive range. It can operate in the industrial, scientific and medical radio band but uses $2.4 \mathrm{GHz}$ in most jurisdictions worldwide. The data transmission rate varies from 20 Kilobits/second in the 868 $\mathrm{MHz}$ frequency band to 250 Kilobits/second in the $2.4 \mathrm{GHz}$ frequency band. It also supports both star and tree networks every network must have one coordinator device, tasked with its creation, the control of its parameters and basic maintenance. Both trees and mesh allows the use of Zigbee network to extend the communication at the network level.

\section{ACQUISITION MODULE}

In the process, the acquisition module is the most significant module in the system will be discussed. This acquisition module is used to control the switching devices connected to the tappings of the primary and secondary side of the transformer and used to sense and transfer the following parameters. They are

\section{Primary Voltage (for Phase A, B and C) \\ 2. Secondary Voltage (for Phase A, B and C) \\ 3. Primary Current (for Phase A, B and C) \\ 4. Secondary Current (for Phase A, B and C) \\ 5. Primary and Secondary Winding Temperature}

In the system, the only one acquisition module is used for the three phase transformer. This acquisition module is used to control all the switching devices and to sense and transfer the parameters of the transformer. The microcontroller in the acquisition module used to control all the switching devices and has ability to sense the parameters of the transformer accurately. This will be the massive task for the controller to handle the switching devices but thinking of cost, this will be highly beneficial and it will be easy to identify the problems in the system.

The design of the switching devices of one phase is connected to the acquisition module is shown in the figure 2.this will be similar to other phases also. As in the figure 2, there are each six switching devices are connected to the tappings of the primary and secondary windings of each phase to form a serial or parallel configuration .this configuration can be achieved by turning ON or OFF the corresponding switching devices as presented in the table I. The implementation of the configuration is used to achieve efficiency or used to reduce the core and copper losses of the transformer. This implementation of the configuration helps in achieving the better efficiency over the conventional transformer. More information about the implementation of the configuration to achieve better efficiency can be read out in [5]. 
In addition to turn the switching devices into ON/OFF conditions, the microcontroller in the module helps in performing the switching operations from series to parallel

Table -I: Switching Device States

\begin{tabular}{|c|l|l|l|l|l|l|}
\hline Configuration & $\mathbf{S W}_{\mathbf{1}}$ & $\mathbf{S W}_{\mathbf{2}}$ & $\mathbf{S W}_{\mathbf{3}}$ & $\mathbf{S W}_{\mathbf{4}}$ & $\mathbf{S W}_{\mathbf{5}}$ & $\mathbf{S W}_{\mathbf{6}}$ \\
\hline Serial & OFF & ON & OFF & OFF & ON & OFF \\
\hline Parallel & ON & OFF & ON & ON & OFF & ON \\
\hline
\end{tabular}

(or vice versa) at zero crossing of current to reduce transient as an effect of switching. Zero crossing of primary voltage is used to trigger the beginning of the sampling process through hardware interruption inside the microcontroller. It also senses the temperature of the windings with the help of the analog temperature sensors. Performing this process, the real time monitoring of the current and voltage signals are done by the microcontroller used for the following purposes
- $\quad$ To determine the winding configurations and timing for switching operations.

- To calculate the true RMS voltages and currents, average power and losses.

- Protection against overloads and transients.

The processes data sent by the microcontroller will be transmitted to the server module through wireless communication.

\section{REMOTE MONITORING}

Remote monitoring of transformers has already been widely known and implemented with various technologies. Many approaches are developed to perform the remote monitoring in the world through the use of network connected to the internet.

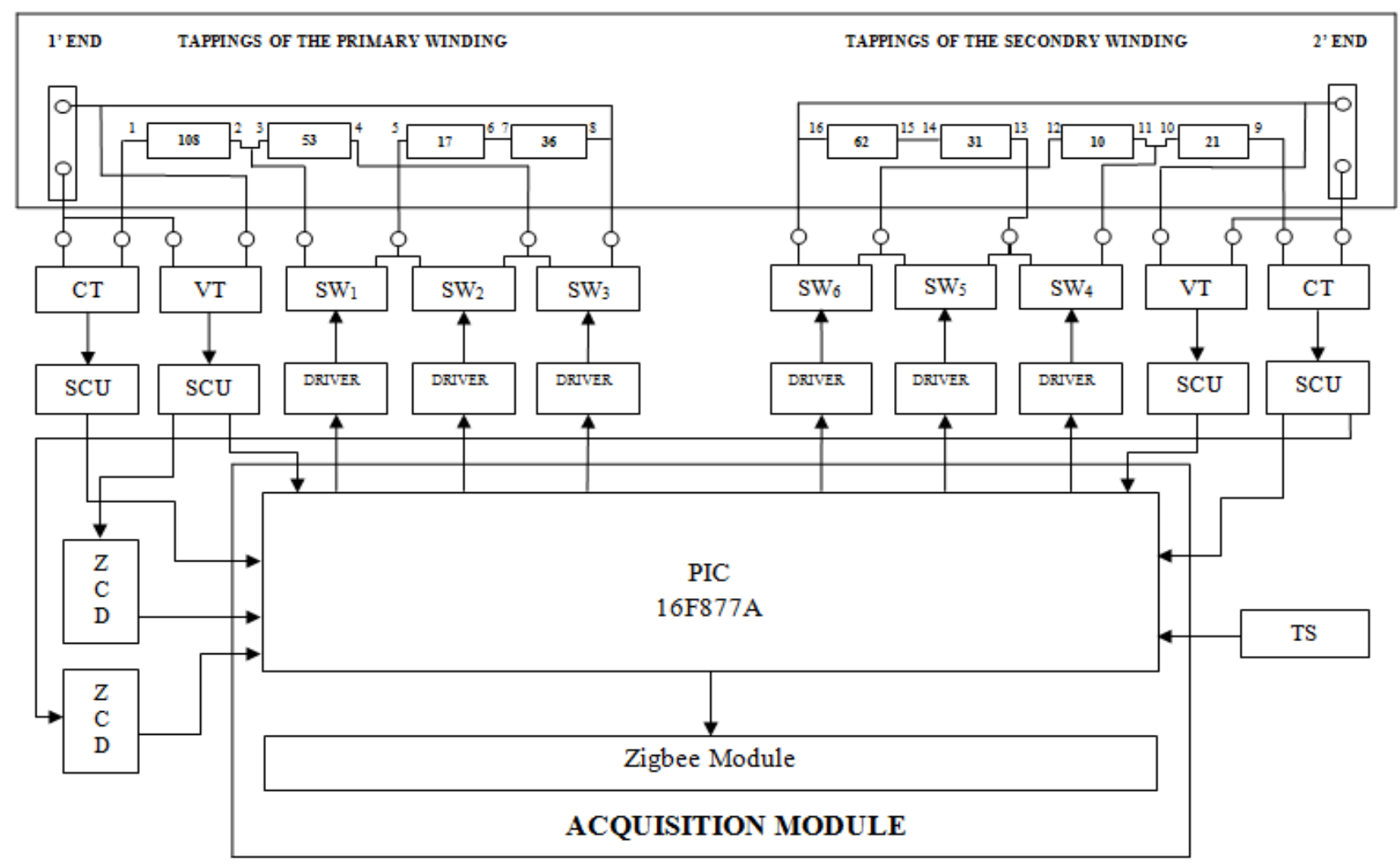

CT : Current Transducer

VT : Voltage Transformer

SGU : Signal Conditioning Unit

ZCD : Zero Crossing Detector

SW : Switching device

TS : Temperature Sensor

Fig- 2: Acquisition Module Connected to One Phase of the Three-Phase 10-kVA Switchable Transformer 
In relation to this project, this was applied to the three-phase 10-kVA switchable distribution transformer. This transformer has been previously developed to show an increase in the efficiency through the use of special configurations of windings called serial and parallel configurations. The serial configuration is highly efficient at low loads with higher number of turns and parallel configuration is highly efficient at high loads with a low number of turns of both the windings of the switchable distribution transformer. These configurations are used to increase efficiency by reducing core and copper losses of the switchable distribution transformer.
This resultant data will be compared with the previous data to know the condition of the transformer.

This monitoring system continuously monitors the conditions of the transformer for 24 hours. The microcontroller in the system has the mechanism to send an alarm or turn OFF the switching devices before overheating occurs. This monitoring system is highly useful in areas where the transformer is far away from the server or from the substation.

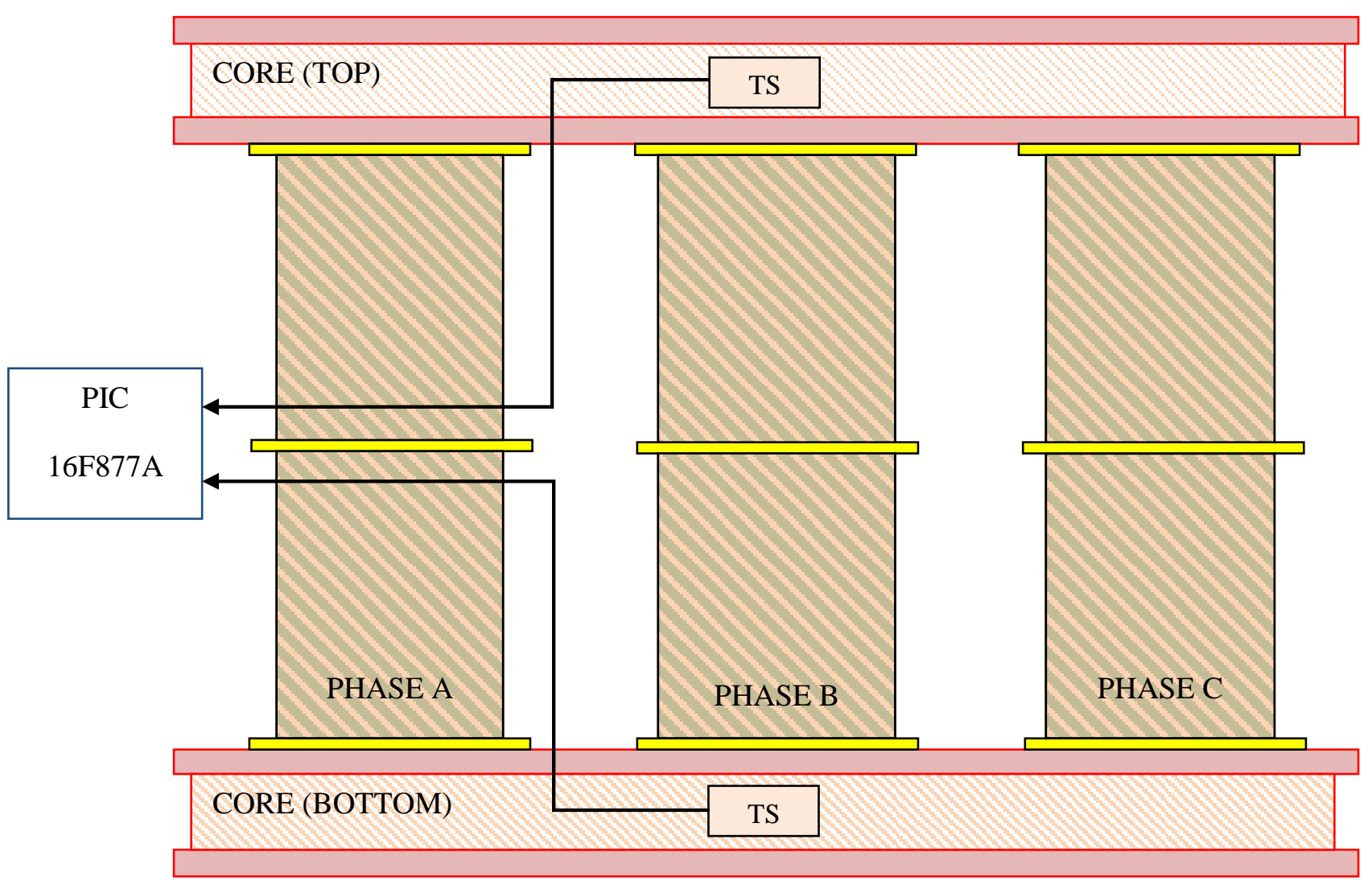

Fig-3: Temperature Monitoring

In relation to this project, we designed a remote monitoring system as shown in the fig. 4 which is based on PIC microcontroller present in the acquisition module used to monitor and control the voltages, currents and temperatures of the switchable distribution transformer and also used to switch ON/OFF the switching devices depends upon the configurations. Each phase data will be monitored for 10 secs by the acquisition module and it will be transmitted to the server module through Zigbee.

The Zigbee has a large coverage area up to $100 \mathrm{~m}$ and has a transmission data rate ranging from $20 \mathrm{Kbps}$ to $250 \mathrm{Kbps}$ with minimum power consumption.

The Radio Frequency (RF) antenna used in the system is tuned for selected frequency for data transmission. On the receiver side, the receiver antenna used in the system converts The RF signal into an electrical signal and acquires the information transmitted by the acquisition module. The resultant data will be displayed in the personal computer.
This system is cost effective and easy to implement in remote areas. This monitoring system uses only one microcontroller as a main controller to monitor the parameters which helps to know the conditions of the transformer. This system uses a very little power consumption and has a long battery life.

\section{SIMULATION RESULT}

This is the simulation result of the remote monitoring system. The software used for the simulation is Proteus on a MPLAB IDE compiler. The parameter of the temperature, current and voltage by the PIC 16F877A microcontroller are monitored from the temperature sensor, current transducer and from the voltage transformer (variable resistors are used instead) and displayed using the $16 \times 2$ alphanumeric LCD display. The LED-BIBY will glow according to the temperature condition 


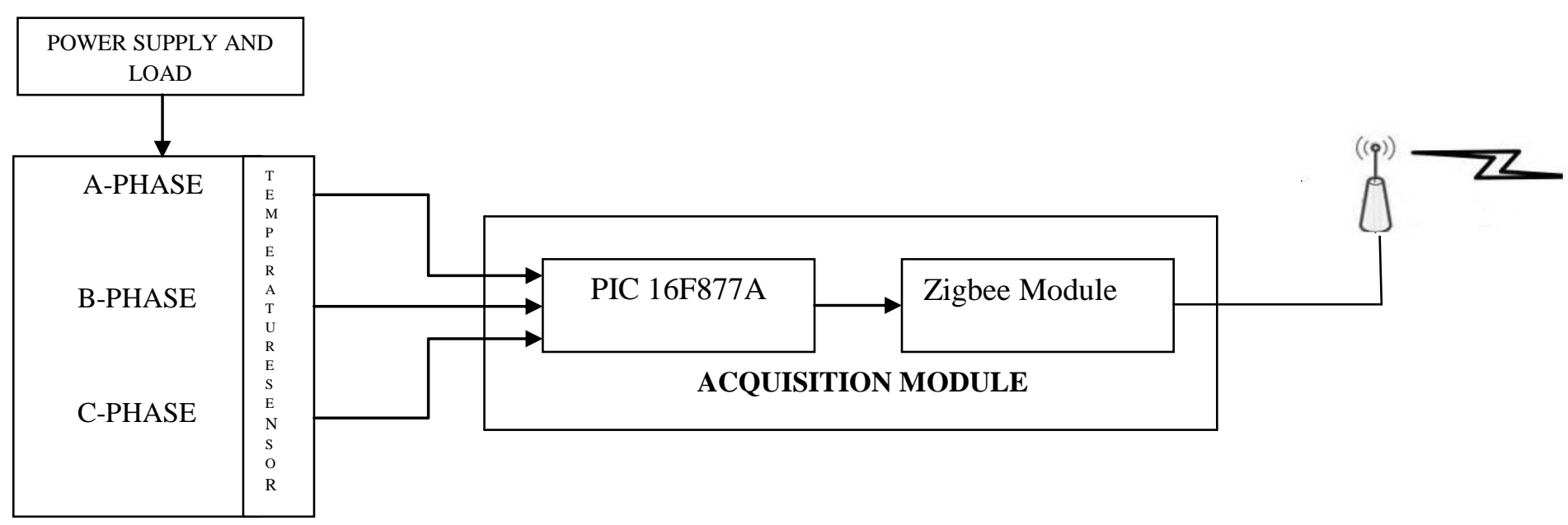

Three-Phase 10-kVA

Switchable Transformer

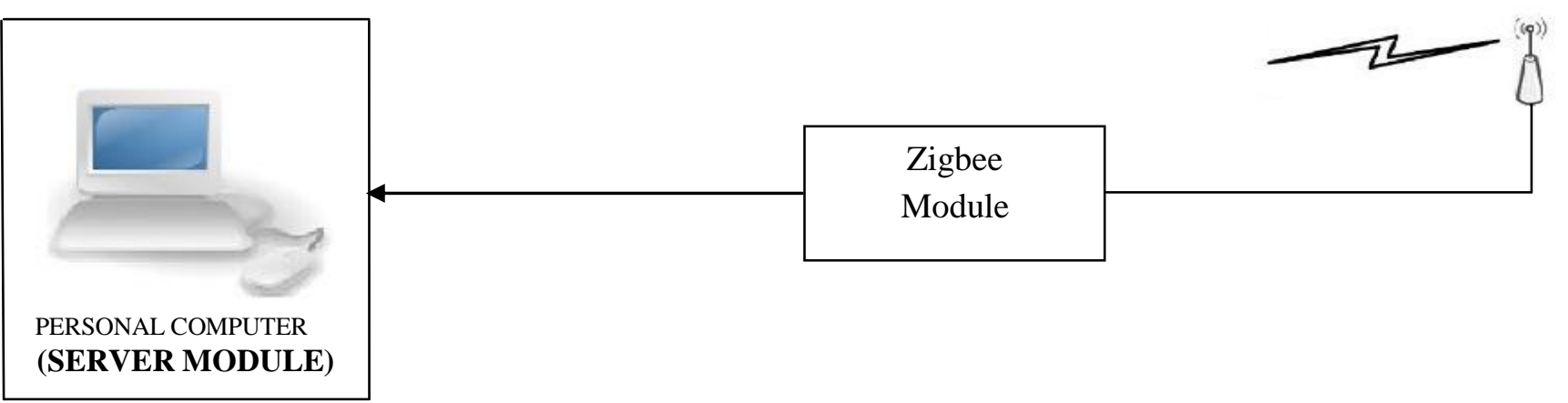

Fig-4: Block Diagram of Remote Monitoring System for a Three-Phase 10-kVA Switchable Transformer

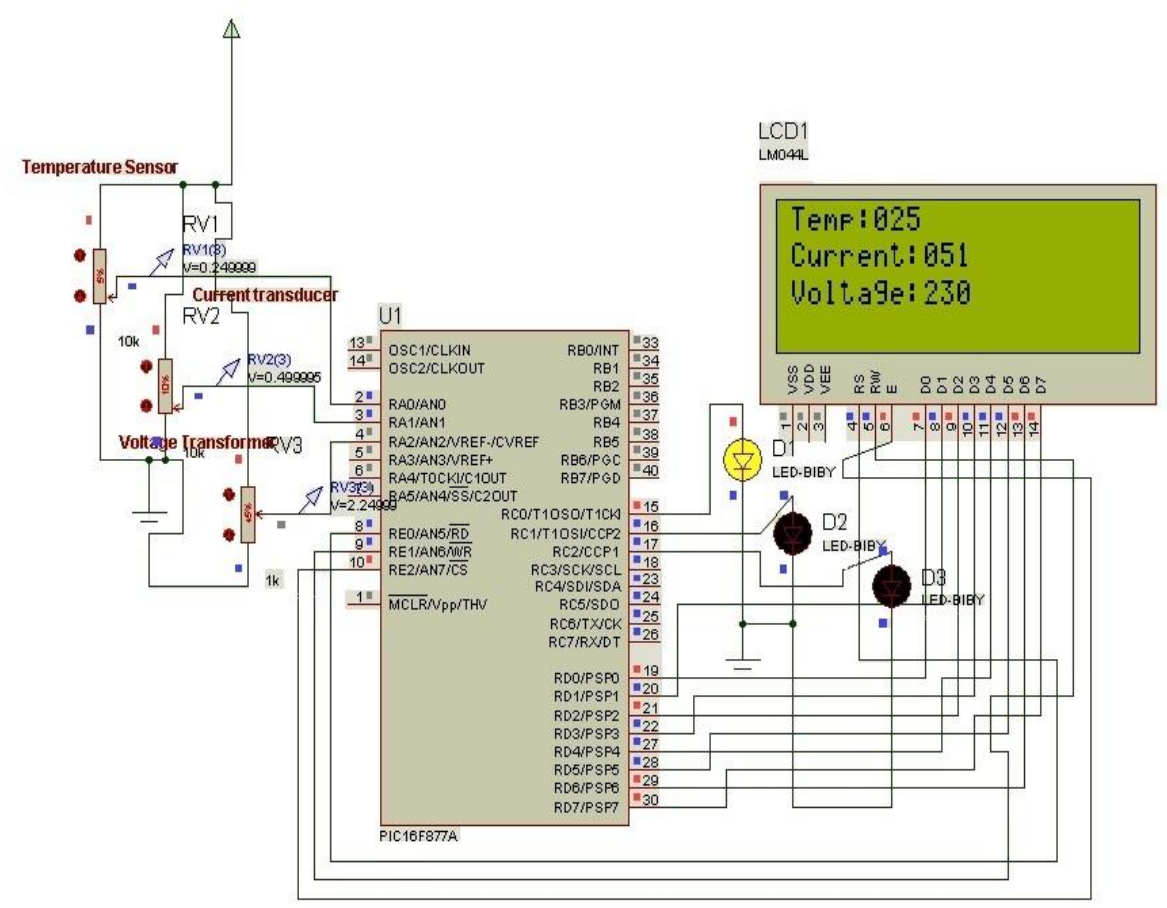

Fig-5: Simulation Result 


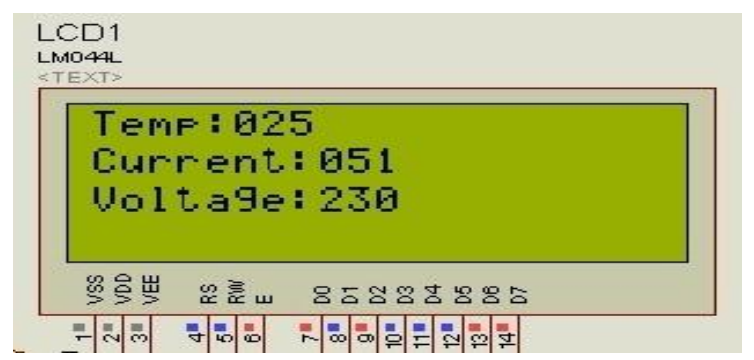

Fig-6: Monitored Parameters

\section{CONCLUSION}

The remote monitoring system that has been developed was useful in understanding the conditions of the transformer. It also enables the operator to monitor the parameters far away from the transformer without the displacement of the crew. This system uses very little power consumption and has a long battery life. This system is cost effective and is easy to operate, to maintain, and to reproduce massively with low cost for applications in the field.

\section{REFERENCES}

[1] H.A.Dharmawan and S. A. M. Ali, "A Compact Remote Monitoring System for a Three-Phase 10-kVA Energy -Efficient Switchable Distribution Transformer," IEEE Transactions on instrument and measurement VOL. 61, NO. 3, march 2012

[2] H. A. Dharmawan and S. A. M. Ali, "A modular approach and controller area network bus implementation in an embedded system of a 3phase $10 \mathrm{kVA}$ energy efficient switchable distribution transformer," in Proc. $5^{\text {th }}$ IEEE Conf. Ind. Electron. Appl., Taichung, Taiwan, June 1517, 2010,pp. 62-67.

[3] Mei-sung,Yu-Lung Ke and He-Yau Kang," Zigbee Wireless Network for Transformer Load Monitoring and Temperatute Sensitivity Analysis" IEEE Industrial Applications Social Annual meeting (IAS), 2011

[4] Abniki, H. Afsharirad, A. Mohseni, F. Khoshkhati, H. Monsef, and P. Sahmsi, "Effective on-line parameters for transformer monitoring and protection," in Proc. NAPS, 2010, pp. 1-5.

[5] H. A. Dharmawan and S. A. M. Ali, "Development of switchable transformer research at University of South Australia," in Proc. Int. Conf. Renew. Energies Power Qual., Valencia, Spain, Apr. 1517, 2009,pp. 1-6.
[6] H. A. Dharmawan and S. A. M. Ali, "Managing distributed temperature measurements in an embedded system of a 3-phase $10 \mathrm{kVA}$ energy efficient switchable distribution transformer employing a modular approach,"in Proc. 2nd Int. Conf. ACT, 2010, pp. 15-20.

[7] F. Poza, P. Marino, S. Otero, and F. Machado, "Programmable electronic instrument for condition monitoring of in-service power transformers,"IEEE Trans. Instrum. Meas., vol. 55, no. 2, pp. 625-634, Apr. 2006.

[8] Dharmawan, H. A. \& Ali, S. A. M., "Design of Switchable Transformer Control System Employing the Micro-controller80C552", International Journal of Applied Electromagnetics and Mechanics, Japan, Vol. 13, pp.99-106, ISBN 1 $586033271,2002$.

[9] Microchip Technology Inc.:PIC16F87X Data Sheet. Microchip Technology Inc. 2003

[10] "Electrical Machinery" book by Dr. P.S.Bimbhra, $7^{\text {th }}$ edition: 2009 , khanna publishers.pg no. 1-159.

\section{BIOGRAPHIES}

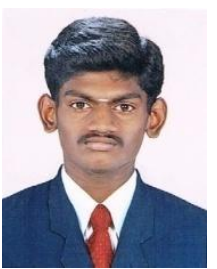

S.Dinesh Kumar received B.E (ECE) from Sri Ramakrishna Engineering College, Coimbatore and currently doing M.E Embedded Systems at Kumaraguru College of Technology, Coimbatore.

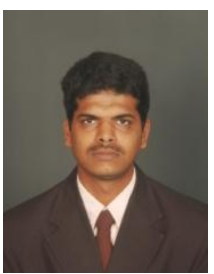

Mr.R.Suresh Kumar received B.E (EEE) from Government College of Technology, Coimbatore and M.E Applied Electronics from PSG College of Technology, Coimbatore. $\mathrm{He}$ is presently working at Kumaraguru College of Technology as assistant Professor. His research and teaching interests include Applied electronics and Embedded systems. He is a member of Indian Society for Technical Education (ISTE).

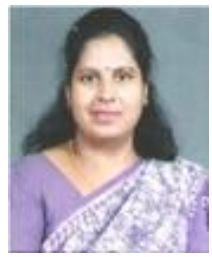

Dr.Rani Thottungal obtained her B.E and M.E degrees from Andhra University and Doctorate from Bharathiar University. She is currently working as Professor and Head in EEE Department at Kumaraguru College of Technology, Coimbatore. Her research interest includes Power System, Power Inverter and Power Quality Issues. 